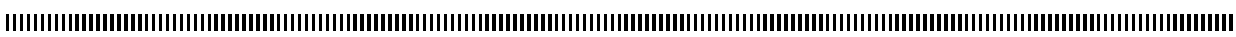

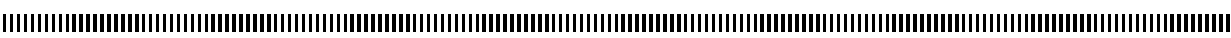

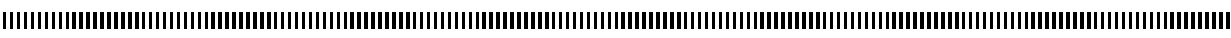

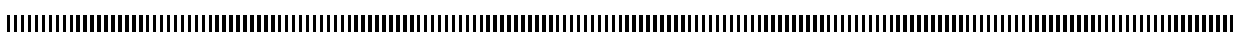

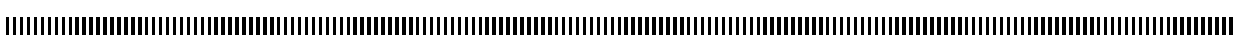
| |

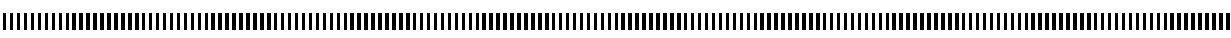

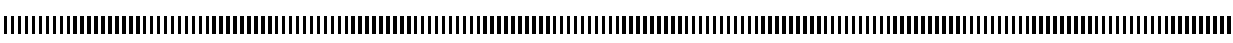
|

\title{
Operating diagram of a flocculation model in the chemostat
}

\author{
Radhouane Fekih-Salem a,c,* - Tewfik Sari b \\ a University of Tunis El Manar, National Engineering School of Tunis, LAMSIN, 1002, Tunis, \\ Tunisia (E-mail: radhouene.fekihsalem@isima.rnu.tn) \\ b ITAP, Univ Montpellier, INRAE, Institut Agro, Montpellier, France \\ (E-mail: tewfik.sari@inrae.fr) \\ c University of Monastir, Higher Institute of Computer Science of Mahdia, 5111, Mahdia, Tunisia \\ * Corresponding author.
}

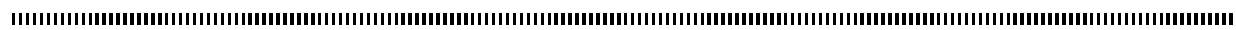

ABSTRACT. The objective of this study is to analyze a model of the chemostat involving the attachment and detachment dynamics of planktonic and aggregated biomass in the presence of a single resource. Considering the mortality of species, we give a complete analysis for the existence and local stability of all steady states for general monotonic growth rates. The model exhibits a rich set of behaviors with a multiplicity of coexistence steady states, bi-stability, and occurrence of stable limit cycles. Moreover, we determine the operating diagram which depicts the asymptotic behavior of the system with respect to control parameters. It shows the emergence of a bi-stability region through a saddle-node bifurcation and the occurrence of coexistence region through a transcritical bifurcation. Finally, we illustrate the importance of the mortality on the destabilization of the microbial ecosystem by promoting the washout of species.

RÉSUMÉ. L'objectif de cette étude est d'analyser un modèle du chémostat impliquant la dynamique d'attachement et de détachement de la biomasse planctonique et agrégée en présence d'une seule ressource. En considérant la mortalité des espèces, nous donnons une analyse complète de l'existence et de la stabilité locale de tous les équilibres pour des taux de croissance monotones. Le modèle présente un ensemble riche de comportements avec multiplicité d'équilibres de coexistence, bi-stabilité et apparition des cycles limites stables. De plus, nous déterminons le diagramme opératoire qui décrit le comportement asymptotique du système par rapport aux paramètres de contrôle. II montre l'émergence d'une région de bi-stabilité via une bifurcation nœud col et l'occurrence d'une région de coexistence via une bifurcation transcritique. Enfin, nous illustrons l'importance de la mortalité sur la déstabilisation de l'écosystème microbien en favorisant le lessivage des espèces.

KEYWORDS : Bi-stability, Bifurcation, Chemostat, Flocculation, Operating diagram

MOTS-CLÉS : Bi-stabilité, Bifurcation, Chémostat, Floculation, Diagramme opératoire

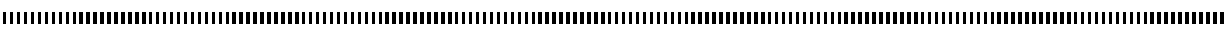




\section{Introduction}

In the culture of microorganisms, the processes of attachment and detachment of bacteria are well known and frequently observed. This phenomenon is manifested either by fixation of microorganisms on support as in the growth of biofilms or simply by an aggregation such as the formation of flocs or granules [9, 21]. In fact, the formation of flocs has a direct impact on growth dynamics, since the access to the substrate is limited for microorganisms within such structures. Nevertheless, it is only recently that they have been explicitly taken into account in mathematical models based on the chemostat (see the monograph [8]).

This flocculation mechanism may explain the coexistence of microbial species when the most competitive species inhibits its own growth by the formation of flocs [7]. In fact, these bacteria in flocs consume less substrate than planktonic bacteria since the attached bacteria have less access to the substrate, given that this access to the substrate is proportional to the outside surface of flocs. An extension of the model [7] has been studied in [5] when the growth rate of isolated bacteria of the most competitive species exhibits an inhibition. In this case, there may be coexistence around a stable limit cycle. The interested reader can refer to $[4,5]$ for a review of the different specific attachment and detachment rates used in the literature.

In this work, we consider the flocculation model of one species introduced in [4]. This model, which has been studied also in $[3,8,15,16]$, is written as follows:

$$
\left\{\begin{aligned}
\dot{S} & =D\left(S_{\text {in }}-S\right)-f(S) u-g(S) v \\
\dot{u} & =\left[f(S)-D_{u}\right] u-a(u+v) u+b v \\
\dot{v} & =\left[g(S)-D_{v}\right] v+a(u+v) u-b v
\end{aligned}\right.
$$

where $S(t)$ is the concentration of the substrate at time $t ; u(t)$ and $v(t)$ are, respectively, the concentrations of planktonic and attached bacteria at time $t ; f(S)$ and $g(S)$ represent, respectively, the growth rates of isolated and attached bacteria; $D$ and $S_{i n}$ are, respectively, the dilution rate and the concentration of the substrate in the feed device; $D_{u}$ and $D_{v}$ represent, respectively, the disappearance rates of planktonic and attached bacteria.

We assume that isolated bacteria can aggregate with isolated bacteria or flocs to form new flocs with a rate $a(u+v) u$, where $a$ is a positive constant, proportional to both the density of isolated bacteria $u$ and the total biomass density $u+v$. Furthermore, the flocs can split and liberate isolated bacteria with rate $b v$, where $b$ is a positive constant, proportional to their density $v$.

Since the size of the flocs is quite large than that of the isolated bacteria, the fraction $\beta$ of attached bacteria which leaves the reactor is lower than that of the isolated bacteria $\alpha$. In [3], the study of this model (1) in the case without mortality has been limited to the biologically interesting case $D_{v} \leq D_{u} \leq D$, where $D_{u}=\alpha D$ and $D_{v}=\beta D, \alpha$ and $\beta$ belong to [0,1]. In fact, the factor $\alpha$ was introduced in [2], see also [1], to model a reactor with biomass attached to the support or to decouple the residence time of solids and the hydraulic residence time $(1 / D)$. In this work, we study the model (1) where $D_{u}$ and $D_{v}$ can be modeled as in $[14,19]$ by:

$$
D_{u}=\alpha D+m_{u}, \quad D_{v}=\beta D+m_{v}
$$

where the non-negative parameters $m_{u}$ and $m_{v}$ representing mortality (or maintenance) rate are taken into consideration. In fact, more realistic models in the engineering and 
biological literature as well as mathematical studies consider the mortality of species that affects the behavior of system and the coexistence of microbial species [10,11, 12, 13, $17,18,22]$. Here, a significant mortality rate of isolated and/or attached bacteria could increase the removal rates $D_{u}$ and/or $D_{v}$ up to values larger than the dilution rate $D$. Therefore, our study will not be restricted to the cases $D_{v} \leq D_{u} \leq D$, as in $[3,4,8,15$, 16], and the cases $D<D_{u}, D<D_{v}$ or $D_{u}<D_{v}$, which are also of biological interest, will be investigated.

For the complete mathematical analysis of model (1), the reader is referred to [6]. Our main objective in this paper is to describe the operating diagram of the model in order to illustrate the behavior of the system according to the control parameters $D$ and $S_{i n}$.

This paper is organized as follows. First, we present in Section 2 some general hypotheses about the growth functions of flocculation model (1). Then, we analyze the existence and the local stability of steady states according to the dilution rate and the disappearance rates of planktonic and attached bacteria. In Section 3, we present the operating diagram in order to show the regions of emergence of multiplicity of positive steady states according to the control parameters. In Section 4, we study the one-parameter bifurcation diagrams and the numerical simulations in order to validate the theoretical analysis of the operating diagram. Finally, conclusions are drawn in the last Section 5.

\section{Hypotheses and model analysis}

We use the following general hypotheses for growth functions $f(S)$ and $g(S)$ :

(H1) $f(0)=g(0)=0$ and $f^{\prime}(S)>0$ and $g^{\prime}(S)>0$ for all $S>0$.

(H2) $f(S)>g(S)$ for all $S>0$.

Assumption (H1) means that the growth can take place if and only if the substrate is present. In addition, the growth rates of isolated and attached bacteria increase with the concentration of substrate. Assumption (H2) means that bacteria in flocs consume less substrate than isolated bacteria.

The first result shows that our model (1) preserves the biological meaning.

Proposition 2.1 For any non-negative initial condition, the solutions of system (1) remain non-negative and positively bounded. In addition, the set

$\Omega=\left\{(S, u, v) \in \mathbb{R}_{+}^{3}: S+u+v \leq \frac{D}{D_{\min }} S_{i n}\right\}$, where $D_{\min }=\min \left(D, D_{u}, D_{v}\right)$,

is positively invariant and is a global attractor for the dynamics (1).

The proofs of all results of this section are detailed in [6]. In what follows, we use the notations:

$$
\begin{gathered}
\varphi(S)=f(S)-D_{u} \quad \text { and } \quad \psi(S)=g(S)-D_{v} \\
U(S):=\frac{\varphi(S)(\psi(S)-b)}{a[\psi(S)-\varphi(S)]} \quad \text { and } \quad V(S):=-\frac{\varphi^{2}(S)(\psi(S)-b)}{a[\psi(S)-\varphi(S)] \psi(S)} \\
H(S):=f(S) U(S)+g(S) V(S) .
\end{gathered}
$$


From (H1), when equations $f(S)=D_{u}, g(S)=D_{v}$ and $\psi(S)=b$ have solutions, they are unique and we define the usual break-even concentrations

$$
\lambda_{u}=f^{-1}\left(D_{u}\right), \quad \lambda_{v}=g^{-1}\left(D_{v}\right) \quad \text { and } \quad \lambda_{b}=\psi^{-1}(b) .
$$

From (H2), if in addition $D_{v} \geq D_{u}$, then $\lambda_{v}>\lambda_{u}$. When equations $f(S)=D_{u}$ or $g(S)=D_{v}$ or $\psi(S)=b$ have no solution, we put $\lambda_{u}=\infty$ or $\lambda_{v}=\infty$ or $\lambda_{b}=\infty$.

\subsection{Existence of steady states}

In order to study the existence of steady states of model (1), we define the interval $I$ by:

$$
I=\left\{\begin{array}{lll}
] \lambda_{u}, \lambda_{v}[ & \text { if } \quad \lambda_{u}<\lambda_{v} \\
] \lambda_{v}, \min \left(\lambda_{u}, \lambda_{b}\right)[ & \text { if } \quad \lambda_{u}>\lambda_{v}
\end{array}\right.
$$

We can state the following result:

Lemme 2.1 Under the assumptions (H1-H2), system (1) has two types of steady states:

1) the washout $E_{0}=\left(S_{i n}, 0,0\right)$, that always exists,

2) a positive steady state, $E_{1}=\left(S^{*}, u^{*}, v^{*}\right)$ with $S^{*}$ solution of equation

$$
D\left(S_{i n}-S^{*}\right)=H\left(S^{*}\right)
$$

where $H$ is given by (3), $u^{*}=U\left(S^{*}\right)$ and $v^{*}=V\left(S^{*}\right)$, where $U$ and $V$ are given by (2). This coexistence steady state exists if and only if $S^{*} \in I$ where I is defined by (4).

The next proposition presents the number of positive steady states of (1).

\section{Proposition 2.2}

- When $D_{u} \leq D_{v}$, then the positive steady state $E_{1}=\left(S^{*}, u^{*}, v^{*}\right)$ exists if and only if $S_{i n}>\lambda_{u}$. If it exists, it is unique.

- When $D_{u}>D_{v}$, then there exists at least one positive steady state in the case $\lambda_{u}<\min \left(\lambda_{v}, S_{i n}\right)$ or $\lambda_{v}<\min \left(\lambda_{u}, \lambda_{b}\right)<S_{i n}$. Generically, the system can have an odd number of positive steady states. When $S_{i n}<\min \left(\lambda_{u}, \lambda_{b}\right)$ and $\lambda_{v}<\lambda_{u}$, then generically the system has no positive steady state or an even number of positive steady states.

\subsection{Stability of steady states}

In this section, we study the local asymptotic stability of each steady state of system (1). Let $J$ be the Jacobian matrix of (1) at $(S, u, v)$, that is given by

$$
J=\left[\begin{array}{ccc}
-D-f^{\prime}(S) u-g^{\prime}(S) v & -f(S) & -g(S) \\
f^{\prime}(S) u & \varphi(S)-a(2 u+v) & -a u+b \\
g^{\prime}(S) v & a(2 u+v) & \psi(S)+a u-b
\end{array}\right]
$$

The stability of the washout steady state is given as follows:

Proposition 2.3 $E_{0}$ is Locally Exponentially Stable (LES) if and only if $S_{i n}<\lambda_{u}$ and $S_{\text {in }}<\lambda_{b}$. 
To analyze the stability of positive steady states, let $J_{1}$ be the Jacobian matrix at $E_{1}=$ $\left(S^{*}, u^{*}, v^{*}\right)$ defined by

$$
J_{1}=\left[\begin{array}{ccc}
-m_{11} & -m_{12} & -m_{13} \\
m_{21} & -m_{22} & a_{23} \\
m_{31} & m_{32} & -m_{33}
\end{array}\right]
$$

where

$$
\left\{\begin{array}{l}
m_{11}=D+f^{\prime}\left(S^{*}\right) u^{*}+g^{\prime}\left(S^{*}\right) v^{*}, \quad m_{12}=f\left(S^{*}\right), \quad m_{13}=g\left(S^{*}\right) \\
m_{21}=f^{\prime}\left(S^{*}\right) u^{*}, \quad m_{22}=a\left(2 u^{*}+v^{*}\right)-\varphi\left(S^{*}\right), \quad a_{23}=b-a u^{*} \\
m_{31}=g^{\prime}\left(S^{*}\right) v^{*}, \quad m_{32}=a\left(2 u^{*}+v^{*}\right) \quad \text { and } \quad m_{33}=b-a u^{*}-\psi\left(S^{*}\right) .
\end{array}\right.
$$

The characteristic polynomial is given by

$$
\begin{gathered}
P(\lambda)=\lambda^{3}+c_{1} \lambda^{2}+c_{2} \lambda+c_{3}, \\
c_{1}=m_{11}+m_{22}+m_{33}, \\
c_{2}=m_{12} m_{21}+m_{13} m_{31}-m_{32} a_{23}+m_{11} m_{22}+m_{11} m_{33}+m_{22} m_{33}, \\
c_{3}=m_{11}\left(m_{22} m_{33}-m_{32} a_{23}\right)+m_{21}\left(m_{12} m_{33}+m_{32} m_{13}\right)+m_{31}\left(m_{12} a_{23}+m_{13} m_{22}\right) .
\end{gathered}
$$

According to Routh-Hurwitz criterion, $E_{1}$ is LES if and only if

$$
c_{1}>0, \quad c_{3}>0 \quad \text { and } \quad c_{4}=c_{1} c_{2}-c_{3}>0
$$

We have the following results:

Lemme 2.2 All $m_{i j}$ are positive for all $i, j=1, \ldots, 3$ with $(i, j) \neq(2,3)$ and we have $c_{1}>0$.

The next lemma shows that the sign of $c_{3}$ is given by the position of the curve of the function $H(\cdot)$ with respect to the line $\delta$ of equation $y=D\left(S_{i n}-S\right)$ (see Fig. 3(b)). More precisely, we give the link between the determinant of the Jacobian matrix $J_{1}$ at $E_{1}=\left(S^{*}, u^{*}, v^{*}\right)$ and $D+H^{\prime}\left(S^{*}\right)$.

Lemme 2.3 One has $c_{3}=-\operatorname{det}\left(J_{1}\right)=-\varphi\left(S^{*}\right)\left(\psi\left(S^{*}\right)-b\right)\left(D+H^{\prime}\left(S^{*}\right)\right)$.

Since the condition $c_{4}>0$ of the Routh-Hurwitz criterion (5) could be unfulfilled, we will study the behavior of flocculation model (1) according to the dilution rate and the disappearance rates of planktonic and attached bacteria. In fact, there exist four cases that must be distinguished:

Case 1: $\quad D_{u} \leq D_{v} \leq D$,

Case 2: $\quad D_{v}<D_{u} \leq D$,

Case 3: $\quad D_{v}<D_{u}$ and $D<D_{u}$,

Case 4: $\quad D_{u} \leq D_{v}$ and $D<D_{v}$.

To determine the local stability of the positive steady state in the first and second cases of (6), we will have need of the following.

Lemme 2.4 In the cases 1 and $2\left(D_{u} \leq D\right.$ and $\left.D_{v} \leq D\right)$, we have $c_{4}>0$.

It was shown in [8], see also [15, 16] that if $D_{u}=D_{v}=D$, then $E_{1}$ exists and is unique and LES if and only if $S_{i n}>\lambda_{u}$. Actually, this result holds in case 1 . 
Proposition 2.4 In the case $1\left(D_{u} \leq D_{v} \leq D\right)$, the positive steady state $E_{1}=\left(S^{*}, u^{*}, v^{*}\right)$ exists if and only if $S_{i n}>\lambda_{u}$. If it exists, it is unique and LES.

The case 2 was solved in [3] where it was shown that the stability depends only on the relative position of the curve of function $y=H(S)$ and the straight line $\delta$ of equation $y=D\left(S_{i n}-S\right)$ that is to say, on the sign of $D+H^{\prime}\left(S^{*}\right)$. More precisely, we have:

Proposition 2.5 Let $E_{1}=\left(S^{*}, u^{*}, v^{*}\right)$ be a positive steady state. Assume that case 2 holds.

1) If $\lambda_{u}<\lambda_{v}$ : $E_{1}$ is LES if $H^{\prime}\left(S^{*}\right)>-D$ and is unstable if $H^{\prime}\left(S^{*}\right)<-D$.

2) If $\lambda_{u}>\lambda_{v}$ : $E_{1}$ is LES if $H^{\prime}\left(S^{*}\right)<-D$ and is unstable if $H^{\prime}\left(S^{*}\right)>-D$.

In the case 3 of (6), when $D<D_{v} \leq D_{u}$ or $D_{v}<D \leq D_{u}, c_{4}$ can change sign by varying the control parameter $S_{i n}$ such that the positive steady state $E_{1}$ could change its behavior without any collision with another steady state [6]. In fact, numerical simulations show the emergence of stable limit cycles by Hopf bifurcations.

In case 4 of (6), we always have $\lambda_{u}<\lambda_{v}$ and $H^{\prime}(S)>0$. Therefore, from Lemma 2.3 , it is deduced that in case 4 of (6) we always have $c_{3}>0$. We were not able to find a set of parameters for which $c_{4}<0$, as in the case 3 of (6) and we conjecture that in this case the positive steady state $E_{1}$ which is unique as soon as it exists, is also LES as soon as it exists.

\section{Operating diagrams}

The operating diagrams show how the system behaves when we vary the two control parameters $S_{i n}$ and $D$ in (1). All other parameters in (1) are fixed, such as growth functions and specific attachment and detachment velocities. In fact, they depend on the nature of the organisms and the substrate introduced into the chemostat. Note that the operating diagrams of flocculation model (1) have not been studied in the existing literature in the generic case where the disappearance rates are distinct.

To determine the various regions in the operating diagrams, we need to define some auxiliary functions that are listed in Table 1 . The domain of definition of the functions $\lambda_{u}, \lambda_{v}$ and $\lambda_{b}$ can easily be established. These functions are increasing in $D$ because the functions $f$ and $g$ are increasing in $S$. However, to define the function $D \mapsto \lambda_{\mathrm{SN}}(D)$ in Table 1 whose curve in the operating diagram corresponds to the saddle-node bifurcation with the appearance of two positive steady states, we consider the case

$$
\begin{cases}\lambda_{v}(D)<\lambda_{u}(D) & \text { for all } D \in\left[0, \bar{D}_{u}[\text { and }\right. \\ \lambda_{u}(D)<\lambda_{b}(D) & \text { for all } D \in\left[0, \bar{D}_{b}[.\right.\end{cases}
$$

Let $\Gamma_{i}, i=u, v, b$, SN, be the respective curves of equations $S_{i n}=\lambda_{i}(D)$. For the specific growth rates (8) and the parameter values in Table 4, the conditions (7) are satisfied, see Fig. 1(a).

In this case (7), the functions $D \mapsto \lambda_{i}(D), i=u, v, b$, tend to infinity as $D$ tends to $\bar{D}_{i}$ defined in Table 1 with $\bar{D}_{b}<\bar{D}_{u}<\bar{D}_{v}$. From Lemma 2.5 in [6], the function $S \mapsto H(S)$ is defined and decreasing on $\left.] \lambda_{v}(D), \lambda_{u}(D)\right]$ for $0 \leq D<\bar{D}_{u}$. In addition, this function $H$ vanishes at $\lambda_{u}(D)$ and tends to infinity as $S$ tends to $\lambda_{v}(D)$ (see Fig. 3(b)). If $\bar{D}_{u} \leq D<\bar{D}_{v}$, then the function $H$ is defined, decreasing on $] \lambda_{v}(D),+\infty[$ and tends to a positive constant as $S$ tends to $+\infty$. If $D \geq \bar{D}_{v}$, then the function $H$ no longer defined since $\lambda_{v}(D)=+\infty$. 
For simplicity, we assume that $H$ is convex as this property is satisfied with the specific growth rates (8). The general case where the function $H$ is not convex can be treated similarly, without added difficulty. In this particular case, the equation $H^{\prime}(S)=-D$ has a unique solution $D \mapsto S_{\mathrm{sN}}(D)$ which is defined and decreasing for $D>-H^{\prime}\left(\lambda_{u}(D)\right)$ and $D<\bar{D}_{v}$ (see Table 1). Note that if $\bar{D}_{u} \leq D<\bar{D}_{v}$, then $H^{\prime}\left(\lambda_{u}(D)\right)=H^{\prime}(+\infty)=0$. Thus, the function $D \mapsto D+H^{\prime}\left(\lambda_{u}(D)\right)$ is increasing from $H^{\prime}\left(f^{-1}\left(m_{u}\right)\right)<0$ for $D=0$ to $\bar{D}_{u}>0$ for $D=\bar{D}_{u}$. Therefore, there exists a unique solution $\left.\bar{D} \in\right] 0, \bar{D}_{u}[$ of equation $D+H^{\prime}\left(\lambda_{u}(D)\right)=0$. In addition, $D>-H^{\prime}\left(\lambda_{u}(D)\right)$ if and only if $D>\bar{D}$. Finally, we may conclude that the functions $D \mapsto S_{\mathrm{SN}}(D)$ and $D \mapsto \lambda_{\mathrm{SN}}(D)$ are defined for $\bar{D}<D<\bar{D}_{v}$.

Table 1 - Notations, auxiliary functions and their domains of definition.

\begin{tabular}{ll}
\hline \hline Notations & Auxiliary functions and domains of definition \\
\hline \multirow{2}{*}{$\lambda_{u}(D)$} & $\begin{array}{l}\lambda_{u}(D)=f^{-1}\left(\alpha D+m_{u}\right) . \\
\text { It is defined for } 0 \leq D<\bar{D}_{u}:=\left(f(+\infty)-m_{u}\right) / \alpha .\end{array}$ \\
\hline$\lambda_{v}(D)$ & $\begin{array}{l}\lambda_{v}(D)=g^{-1}\left(\beta D+m_{v}\right) . \\
\text { It is defined for } 0 \leq D<\bar{D}_{v}:=\left(g(+\infty)-m_{v}\right) / \beta .\end{array}$ \\
\hline \multirow{2}{*}{$\lambda_{b}(D)$} & $\begin{array}{l}\lambda_{b}(D)=g^{-1}\left(\beta D+m_{v}+b\right) . \\
\text { It is defined for } 0 \leq D<\bar{D}_{b}:=\left(g(+\infty)-m_{v}-b\right) / \beta .\end{array}$ \\
\hline \multirow{2}{*}{$S=S_{\mathrm{SN}}(D)$} & $\begin{array}{l}S=S_{\mathrm{SN}}(D) \text { is the unique solution of equation } H^{\prime}(S)=-D . \\
\text { It is defined for } \bar{D} \leq D<\bar{D}_{v} .\end{array}$ \\
\hline \multirow{2}{*}{$\lambda_{\mathrm{SN}}(D)$} & $\begin{array}{l}\lambda_{\mathrm{SN}}(D)=H\left(S_{\mathrm{SN}}(D)\right) / D+S_{\mathrm{SN}}(D) . \\
\text { It is defined for } \bar{D} \leq D<\bar{D}_{v} .\end{array}$ \\
\hline \hline
\end{tabular}

In order to illustrate the operating diagram, we considered the parameter values provided in Table 4 with the growth rates $f$ and $g$ of Monod-type:

$$
f(S)=\frac{m_{1} S}{k_{1}+S} \quad \text { and } \quad g(S)=\frac{m_{2} S}{k_{2}+S},
$$

where $m_{i}$ denotes the maximum growth rate and $k_{i}$ the Michaelis-Menten constant, $i=$ 1,2. For these specific growth rates (8), the various functions defined in Table 1 are listed in Table 5, except the functions $S_{\mathrm{SN}}$ and $\lambda_{\mathrm{SN}}$ whose expressions are quite complex.

For the set of parameters mentioned in Table 4, the numerical simulations show that the condition $c_{4}>0$ of the Routh-Hurwitz criterion is satisfied in the case (7). However, this condition may not be satisfied for another set of parameters where the positive steady state can change behavior by a Hopf bifurcation with the emergence of a stable limit cycle. In this case, the analysis of the operating diagram is the subject of on-going investigations.

From Lemma 2.3, the positive steady state $E_{1}=\left(S^{*}, u^{*}, v^{*}\right)$ satisfies $c_{3}\left(S^{*}\right)>0$ since $\varphi\left(S^{*}\right)<0, \psi\left(S^{*}\right)<b$ and $H^{\prime}\left(S^{*}\right)<-D$ in this case (7). By Lemma 2.2, we conclude that all the conditions of the Routh-Hurwitz criterion are satisfied, that is, $E_{1}$ is LES as long as it exists.

However, the positive steady state $E_{2}=\left(S^{* *}, u^{* *}, v^{* *}\right)$ defined by $H^{\prime}\left(S^{* *}\right)>-D$ is unstable as long as it exists since $c_{3}\left(S^{* *}\right)<0$ (see Fig. 3(b)). To establish the operating diagram, we summarize the existence and local stability of all steady states in Table 2 .

From Table 2 and the previous results, we see that the conditions of existence and stability of all steady states depend only on the functions $\lambda_{\mathrm{SN}}$ and $\lambda_{u}$ while $\lambda_{v}$ and $\lambda_{b}$ play 
Table 2 - Conditions of existence and stability of steady states in the case (7).

\begin{tabular}{lll}
\hline \hline Steady state & Existence & Local stability \\
\hline$E_{0}$ & always exists & $S_{i n}<\lambda_{u}(D)$ \\
$E_{1}$ & $\lambda_{\mathrm{SN}}(D)<S_{i n}$ & always stable \\
$E_{2}$ & $\lambda_{\mathrm{SN}}(D)<S_{i n}<\lambda_{u}(D)$ & always unstable \\
\hline \hline
\end{tabular}

no role in the operating diagram. On the other hand, $\Gamma_{u}$ and $\Gamma_{\mathrm{SN}}$ separate the operative plan $\left(D, S_{\text {in }}\right)$ at most in three regions, denoted $\mathcal{I}_{0}, \mathcal{I}_{1}$ and $\mathcal{I}_{2}$ (see Fig. 1(b)). Thus, we can state the next result.

Proposition 3.1 The existence and local stability of steady states $E_{0}, E_{1}$ and $E_{2}$ in the regions $\mathcal{I}_{0}, \mathcal{I}_{1}$ and $\mathcal{I}_{2}$ of the operating diagram shown in Fig. $1(b)$ are given in Table 3.

Table 3 - Existence and local stability of steady states according to the regions in the operating diagram of Fig. 1(b). The letter S (resp. U) means stable (resp. unstable). Absence of letter means that the corresponding steady state does not exist.

\begin{tabular}{lccccc}
\hline \hline Condition & Region & Color & $E_{0}$ & $E_{1}$ & $E_{2}$ \\
\hline$S_{\text {in }}<\lambda_{\mathrm{SN}}(D)$ & $\left(D, S_{\text {in }}\right) \in \mathcal{I}_{0}$ & cyan & $\mathrm{S}$ & & \\
$\lambda_{\mathrm{SN}}(D)<S_{\text {in }}<\lambda_{u}(D)$ & $\left(D, S_{i n}\right) \in \mathcal{I}_{1}$ & green & $\mathrm{S}$ & $\mathrm{S}$ & $\mathrm{I}$ \\
$\lambda_{u}(D)<S_{\text {in }}$ & $\left(D, S_{i n}\right) \in \mathcal{I}_{2}$ & red & $\mathrm{I}$ & $\mathrm{S}$ & \\
\hline \hline
\end{tabular}

The transition from the region $\mathcal{I}_{0}$ to the region $\mathcal{I}_{1}$ by the curve $\Gamma_{\mathrm{SN}}$ (in green) corresponds to a saddle-node bifurcation with the appearance of two positive steady states $E_{1}$ which is LES and $E_{2}$ which is unstable. The transition from the region $\mathcal{I}_{1}$ to the region $\mathcal{I}_{2}$ by the curve $\Gamma_{u}$ (in red) corresponds to a transcritical bifurcation when the unstable steady state $E_{2}$ disappears and $E_{0}$ becomes unstable.

Fig. 2 shows the reduction of the bi-stability region (in green) $\mathcal{I}_{1}$ and the coexistence region (in red) $\mathcal{I}_{2}$ by increasing the value of the mortality parameter of the isolated bacteria while the washout region (in cyan) $\mathcal{I}_{0}$ increases. These operating diagrams show that the coexistence and the maintenance of the least competitive species depend on the biological parameters of natural mortality where there can be wash out of bacteria and destabilization of the microbial ecosystem. Hence, the importance of considering these parameters in mathematical models where the behavior of the system is very sensitive to the effect of the natural death of bacteria. Moreover, the control parameter values should be chosen in the region $\mathcal{I}_{2}$ in order to protect the coexistence of two microbial species in this process. However, an appropriate initial condition should be chosen to achieve a good operation of the process when the operating parameters are in the region $\mathcal{I}_{1}$. 

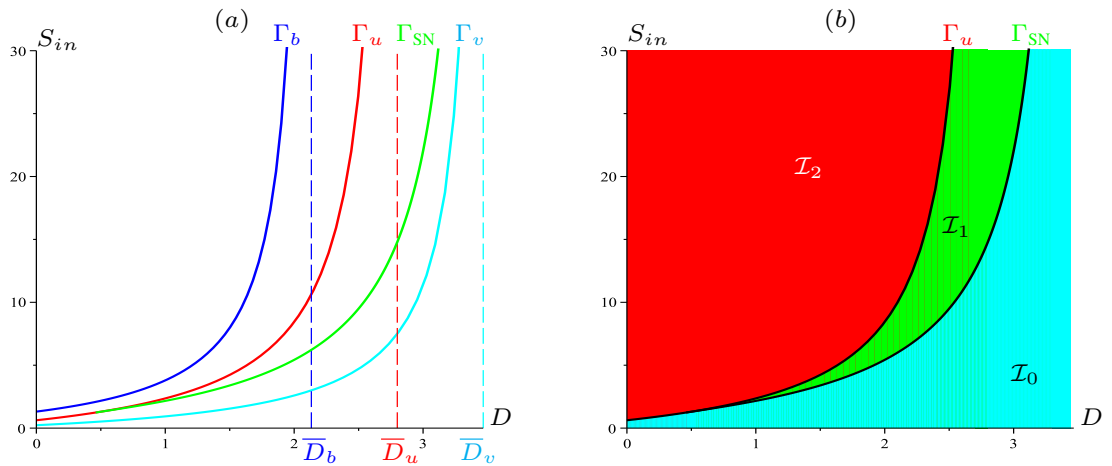

Figure 1 - Case (7): (a) the three curves $\Gamma_{v}, \Gamma_{u}$ and $\Gamma_{b}$ do not intersect and the curves $\Gamma_{u}$ and $\Gamma_{\mathrm{SN}}$ intersect in $\left(\bar{D}, \bar{S}_{i n}\right)=(0.46,1.239)$. (b) The corresponding operating diagram of (1).
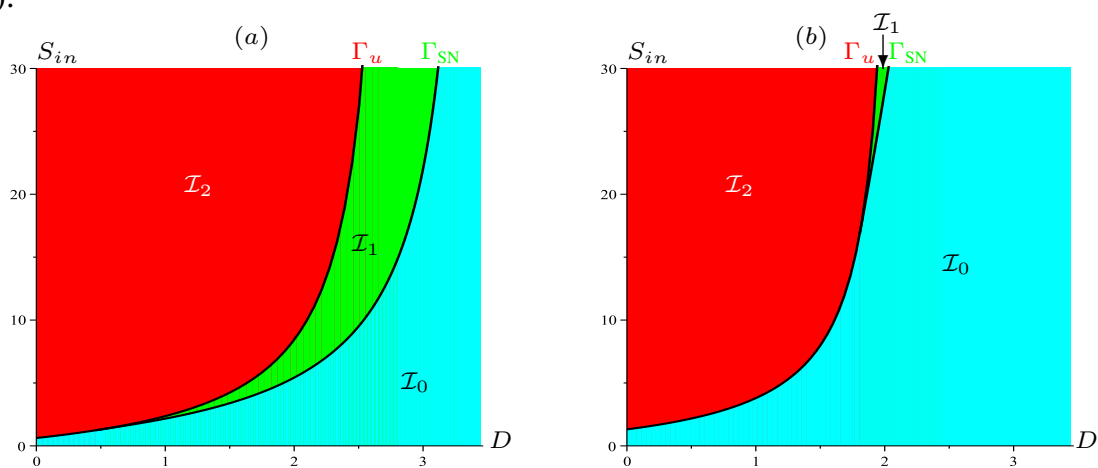

Figure 2 - Effect of mortality on the operating diagram: (a) $m_{u}=0.7$ (b) $m_{u}=50$.

\section{The one-parameter bifurcation diagrams and numerical simulations}

Our aim in this section is to study the behavior of system (1) when only the parameter $S_{i n}$ is varying and all other parameters are fixed. In what follows, we choose $D=2$ and the other parameter values are provided in Table 4. First, we represent in Fig. 3(b) the existence and stability of the steady states when $\left(D, S_{i n}\right)=(2,6)$ which corresponds to the diamond point in the bi-stability region $\mathcal{I}_{1}$ of the operating diagram in Fig. 3(a). In this case, the positive steady states

$$
E_{1} \simeq(3.5,0.583,1.699) \quad \text { and } \quad E_{2} \simeq(5.621,0.198,0.117)
$$

are given by the intersection of the line $\delta$ of equation $y=D\left(S_{i n}-S\right)$ and the curve of the function $H$. In all figures, we have chosen the red color for LES steady states and blue color for unstable steady states. For $D=2$, we have

$$
\lambda_{v}(2) \simeq 2.591<\lambda_{u}(2) \simeq 8.437<\lambda_{b}(2)=43.5 .
$$

Fig. 3(b) shows that $c_{4}\left(S^{*}\right)>0$ for all $\left.S^{*} \in\right] \lambda_{v}(2), \lambda_{u}(2)[$. Note that the curve of the function $H$ is tangent to the line $\delta$ of equation $y=D\left(S_{i n}^{\mathrm{SN}}-S\right)$ when $S=S_{\mathrm{SN}}(2) \simeq 4.338$ such that

$$
S_{i n}^{\mathrm{SN}}=\lambda_{\mathrm{SN}}(2)=\frac{1}{2} H\left(S_{\mathrm{SN}}(2)\right)+S_{\mathrm{SN}}(2) \simeq 5.426 \text {. }
$$



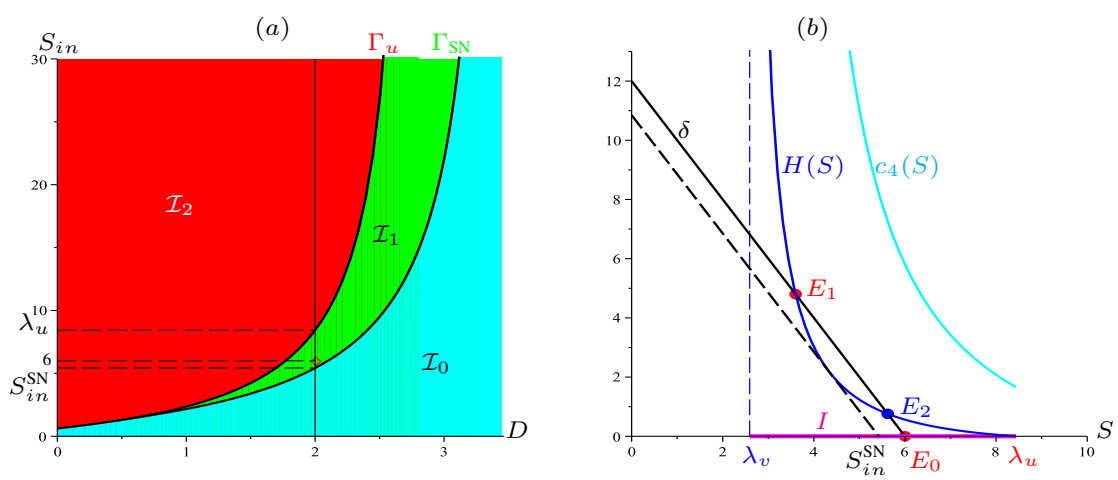

Figure 3 - (a) The operating diagram of (1) when case (7) holds. (b) The existence and stability of steady states corresponding to the diamond point $\left(D, S_{\text {in }}\right)=(2,6) \in \mathcal{I}_{1}$.

Fig. 4(a) shows the coexistence between the two species for an initial condition $S(0)=S^{* *}+\varepsilon, u(0)=u^{* *}+\varepsilon, v(0)=u^{* *}+\varepsilon$, where $\varepsilon=10^{-3}$ and the components of $E_{2}$ are given in (9). The solution of model (1) converges toward the coexistence steady state $E_{1}$ which is LES. Fig. 4(b) shows the extinction of two species for an initial condition $S(0)=S^{* *}-\varepsilon, u(0)=u^{* *}-\varepsilon, v(0)=u^{* *}-\varepsilon$ and the convergence toward the washout steady state $E_{0}=(6,0,0)$ which is LES.

(a)

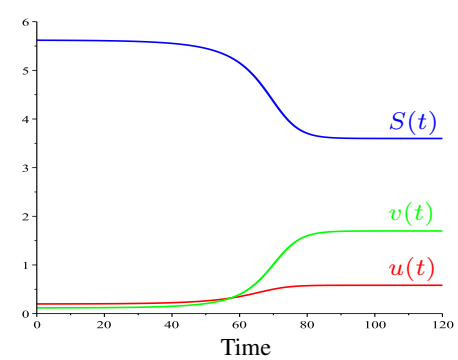

(b)

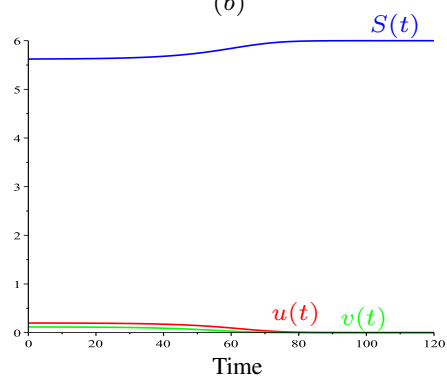

Figure 4 - The system exhibits a bi-stability: according to the initial condition, there is either (a) coexistence of the two species (convergence toward $E_{1}$ ) or (b) washout of the two species (convergence toward $E_{0}$ ).

Fig. 5 illustrates the trajectories over time in three-dimensional space $(S, u, v)$ for several positive initial conditions. For $\left(D, S_{i n}\right)=(2,6) \in \mathcal{I}_{1}$, Fig. 5(a) shows the bistability with two basins of attraction, one toward the washout steady state $E_{0}$ and the other toward the coexistence steady state $E_{1}$ which are stable nodes. These two basins are separated by the stable manifold of saddle point $E_{2}$. For $\left(D, S_{i n}\right)=(2,9) \in \mathcal{I}_{2}$, the numerical simulations can show the global convergence toward the positive steady state $E_{1}=(3.07,0.77,5.15)$ from any positive initial condition (see Fig. 5(b)).

Finally, we study the one-parameter bifurcation diagram for system (1) as the control parameter $S_{i n}$ varies while $D$ is fixed $(D=2)$. Fig. 6 shows a saddle-node bifurcation between $E_{1}$ and $E_{2}$ at the cyan circle point for $S_{i n}=S_{i n}^{\mathrm{SN}} \simeq 5.426$. Then, the washout steady state $E_{0}$ loses its stability by a transcritical bifurcation with $E_{2}$ at the green diamond point for $S_{i n}=\lambda_{u} \simeq 8.437$. 
(a)

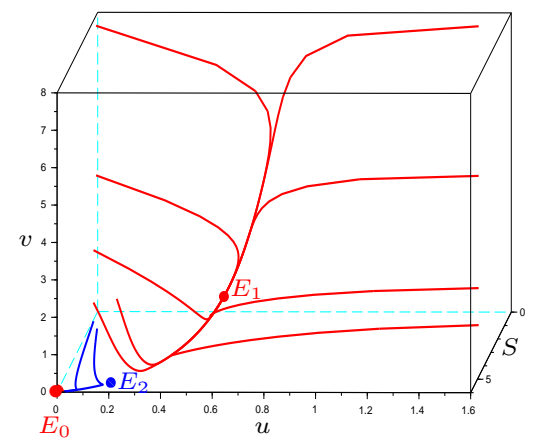

(b)

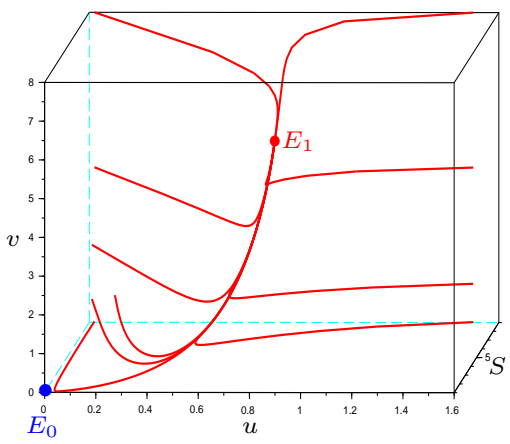

Figure 5 - The trajectories of system (1) in three-dimensional space $(S, u, v)$ (a) the bistability of $E_{0}$ and $E_{1}$ when $\left(D, S_{i n}\right)=(2,6) \in \mathcal{I}_{1}$. (b) Global convergence to $E_{1}$ when $\left(D, S_{\text {in }}\right)=(2,9) \in \mathcal{I}_{2}$.

(a)

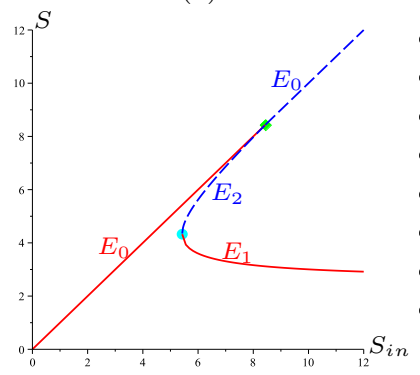

(b)

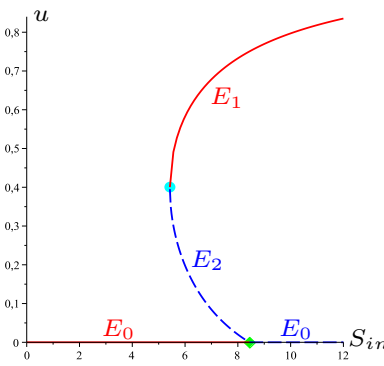

(c)

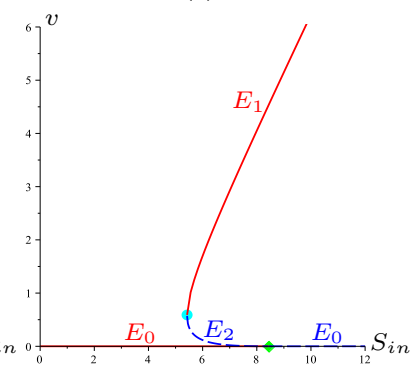

Figure 6-One-parameter bifurcation diagrams for fixed $D=2$ showing the effect on the components $S, u$ and $v$ of all steady states as $S_{i n}$ varies. Blue dashed curves corresponds to unstable steady states and red solid curves to the stable steady states. The green solid diamonds represent the transcritical bifurcations of $E_{0}$ and $E_{2}$ while the cyan solid circles represent the saddle-node bifurcations of $E_{1}$ and $E_{2}$.

\section{Conclusion}

In this work, we have analyzed mathematically and through numerical simulations a model of the chemostat where one species is present in two forms, isolated and attached with the presence of a single resource. The new feature was that maintenance terms are added to removal rates in order to give a complete analysis of the flocculation model (1). The mathematical analysis and numerical simulations show that the model reveals a very rich set of possible behaviors: multiplicity of coexistence steady states, bi-stability and occurrence of stable limit cycles due to Hopf bifurcations.

The operating diagram shows the occurrence of the bi-stability region with multiplicity of coexistence steady states that can bifurcate through saddle-node bifurcations or transcritical bifurcations. However, the bi-stability could occur in the classic chemostat model [20] only when the growth rate is non-monotonic. Furthermore, the operating diagrams show the effect of mortality of the planktonic bacteria on the reduction of the bi-stability and the coexistence regions by promoting the washout of the species and the destabilization of the microbial ecosystem. The one-parameter bifurcation diagram shows the effect of the control parameter $S_{i n}$ on the behavior of the system. 
Our work highlights the common effects of the flocculation phenomenon and mortality to explain the coexistence around a stable positive steady state or a stable limit cycle. In fact, the aggregation of isolated bacteria plays the role of an "ecological niche" that protects microorganisms and allows to avoid the attraction basin of the washout steady state and therefore prevents the species extinction. Thus, the flocculation and mortality are antagonist phenomena: flocculation protects the bacteria while decay tends to wash out them. These two mechanisms can compensate at bistability or create periodic solutions since the trajectories of the system are bounded. This theoretical message explains and reinforces the experimental observations about the positive effect of the flocculation on the coexistence of species and the negative effect of mortality on the destabilization of the system.

Acknowledgments. The first author thanks the LAMSIN (LR-99-ES20), ENIT, University of Tunis El Manar and the French national research institute INRIA for their financial support to participate to CARI 2018 at Stellenbosch University in South Africa, where part of this work was presented. The authors thank the Euro-Mediterranean research network TREASURE (http: //www.inra.fr/treasure).

\section{A. Parameters used in numerical simulations}

Table 4 - Parameter values used for (1) when the growth rates $f$ and $g$ are given by $(8)$.

\begin{tabular}{lllllllllll}
\hline Parameter & $\begin{array}{l}m_{1} \\
\left(h^{-1}\right)\end{array}$ & $\begin{array}{l}k_{1} \\
(g / l)\end{array}$ & $\begin{array}{l}m_{2} \\
\left(h^{-1}\right)\end{array}$ & $\begin{array}{l}k_{2} \\
(g / l)\end{array}$ & $\begin{array}{l}a \\
(l / h / g)\end{array}$ & $\begin{array}{l}b \\
\left(h^{-1}\right)\end{array}$ & $\alpha$ & $\beta$ & $\begin{array}{l}m_{u} \\
\left(h^{-1}\right)\end{array}$ & $\begin{array}{l}m_{v} \\
\left(h^{-1}\right)\end{array}$ \\
\hline $\begin{array}{l}\text { Figs. 1, 3, 4, 5, 6 } \\
\text { Fig. 2 }\end{array}$ & 3.5 & 2.5 & 3 & 1.5 & 1 & 1 & 1 & 0.75 & $\begin{array}{l}0.7 \\
50\end{array}$ & 0.4 \\
\hline
\end{tabular}

\section{B. Auxiliary functions in the case of specific growth rates (8)}

Table 5 - Auxiliary functions and their domains of definition in the case of specific growth rates given by (8).

\begin{tabular}{ll}
\hline \hline Auxiliary functions & Domains of definition \\
\hline$\lambda_{u}(D)=\frac{k_{1}\left(\alpha D+m_{u}\right)}{m_{1}-\alpha D-m_{u}}$ & $0 \leq D<\bar{D}_{u}=\frac{m_{1}-m_{u}}{\alpha}$ \\
$\lambda_{v}(D)=\frac{k_{2}\left(\beta D+m_{v}\right)}{m_{2}-\beta D-m_{v}}$ & $0 \leq D<\bar{D}_{v}=\frac{m_{2}-m_{v}}{\beta}$ \\
$\lambda_{b}(D)=\frac{k_{2}\left(\beta D+m_{v}+b\right)}{m_{2}-\beta D-m_{v}-b}$ & $0 \leq D<\bar{D}_{b}=\frac{m_{2}-m_{v}-b}{\beta}$ \\
\hline \hline
\end{tabular}




\section{References}

[1] B. Benyahia, T. SARi, B. Cherki, J. Harmand, "Bifurcation and stability analysis of a two step model for monitoring anaerobic digestion processes", J. Process Control, vol. 22, 2012, 1008-1019.

[2] O. Bernard, Z. Hadj-Sadok, D. Dochain, A. Genovesi, J-P. Steyer, "Dynamical model development and parameter identification for an anaerobic wastewater treatment process", Biotechnol. Bioeng., vol. 75, 2001, 424-438.

[3] R. FEKIH-SALEM, "Modèles mathématiques pour la compétition et la coexistence des espèces microbiennes dans un chémostat", PhD thesis, UM2-UTM, 2013.

[4] R. Fekih-SAlem, J. HARmand, C. Lobry, A. RApaport, T. SARI, "Extensions of the chemostat model with flocculation", J. Math. Anal. Appl., vol. 397, 2013, 292-306.

[5] R. FeKiH-SAlem, A. RAPAPORT, T. SARI, "Emergence of coexistence and limit cycles in the chemostat model with flocculation for a general class of functional responses", Appl. Math. Modell., vol. 40, 2016, 7656-7677.

[6] R. FeKIH-SAlEm, T. SARI, "Properties of the chemostat model with aggregated biomass and distinct removal rates", SIAM J. Appl. Dyn. Syst., vol. 18, 2019, 481-509.

[7] B. HAEGEMAN, A. RAPAPORT, "How flocculation can explain coexistence in the chemostat", J. Biol. Dyn., vol. 2, 2008, 1-13.

[8] J. HARMAnd, C. Lobry, A. RAPAPORT, T. SARI, "The Chemostat: Mathematical Theory of Microorganism Cultures", Chemical Eng. Ser., Chemostat Bioprocesses Set, Wiley, New York, 2017.

[9] IWA TASk Group on Biofilm Modeling, "Mathematical modeling of biofilms", IWA publishing, 2006.

[10] D. Jones, H.V. Kojouharov, D. Le, H. Smith, "Bacterial wall attachment in a flow reactor", SIAM J. Appl. Math., vol. 62, 2002, 1728-1771.

[11] D. Jones, H. V. Kojouharov, D. Le, H. Smith, "The Freter model: A simple model of biofilm formation", J. Math. Biol., vol. 47, 2003, 137-152.

[12] B.W. KooI, M.P. BoER, "Chaotic behaviour of a predator-prey system in the chemostat", Dyn. Contin. Discrete Impulse Syst. Ser. B App. Algorithms, vol. 10, 2003, 259-272.

[13] B. LI, Y. KUANG, "Simple food chain in a chemostat with distinct removal rates", J. Math. Anal. Appl., vol. 242, 2000, 75-92.

[14] S. Marsili-Libelli, S. Beni, "Shock load modelling in the anaerobic digestion process", Ecol. Model., vol. 84, 1996, 215-232.

[15] A. RAPAPORT, "Properties of the chemostat model with aggregated biomass", Eur. J. Appl. Math., vol. 29, 2018, 972-990.

[16] T. SARI, R. FeKIH-SAlem, "Analysis of a model of flocculation in the chemostat", Proceedings of the 8th conference on Trends in Applied Mathematics in Tunisia, Algeria, Morocco, 2017, 75-80.

[17] T. SARI, J. HARMAND, “A model of a syntrophic relationship between two microbial species in a chemostat including maintenance", Math. Biosci., vol. 275, 2016, 1-9.

[18] T. SARI, M.J. WADE, "Generalised approach to modelling a three-tiered microbial foodweb", Math. Biosci., vol. 291, 2017, 21-37.

[19] S. Shen, G. C. Premier, A. Guwy, R. Dinsdale, "Bifurcation and stability analysis of an anaerobic digestion model”, Nonlinear Dyn., vol. 48, 2007, 391-408.

[20] H.L. Smith, P. Waltman, "The Theory of the Chemostat: Dynamics of Microbial Competition", Cambridge University Press, Cambridge, UK, 1995. 
[21] D.N. Thomas, S.J. Judd, N. FAwcetT, "Flocculation modelling: a review", Water Res., vol. 33, 1999, 1579-1592.

[22] A. Xu, J. Dolfing, T.P. Curtis, G. Montague, E. Martin, "Maintenance affects the stability of a two-tiered microbial 'food chain'?”, J. Theor. Biol., vol. 276, 2011, 35-41. 